\title{
A precision piezodriven micropositioner mechanism with large travel range
}

\author{
S. H. Chang a) and B. C. Du \\ Department of Mechanical Engineering, National Taiwan University, Taipei, Taiwan 10617, \\ Republic of China
}

(Received 16 September 1997; accepted for publication 27 January 1998)

\begin{abstract}
A micropositioning stage with large travel range has been designed and built. The stage combines a piezoelectric driving element, flexure pivoted multiple Scott-Russell linkage, and a parallel guiding spring. Quality engineering techniques are used to optimize the configuration of the device in order to achieve the maximum displacement gain and the minimum angular deviation. A simple open-loop compensator is applied to reduce the hysteresis of the dynamic response of the stage. The experiment shows that the stage achieved a vacuum-compatible device with a travel of greater than $100 \mu \mathrm{m}$, a resolution of $0.04 \mu \mathrm{m}$, and an angular deviation of less than $31.1 \mu \mathrm{rad}$. The first natural frequency of the stage is $80 \mathrm{~Hz}$ and the settling time is approximately $50 \mathrm{~ms}$. Compared with the uncontrolled condition, the controlled hysteresis is reduced significantly. () 1998 American Institute of Physics. [S0034-6748(98)05204-6]
\end{abstract}

\section{INTRODUCTION}

Recently, micropositioning has become a key technology for many fields, such as optical and electron microscopy, $\mathrm{x}$-ray lithography, mask alignment, and electron beam microstructuring. These fields entail an increasing demand for precision positioning devices providing up to $100 \mu \mathrm{m}$ travel range, with micron resolution, and minimum lateral and tilting motion at wide dynamic operating range. In addition, these devices should be vacuum-compatible and able to be remotely activated in order to be isolated from external disturbances. A piezoelectric transducer (PZT) translator can offer high resolution and linear motion. Nevertheless, to meet the goal of long travel range, an optimized guiding and magnifying mechanism is specifically necessary for small electrically induced piezoelectric strain.

Furukawa, Mizuno and Terada ${ }^{1}$ designed a mechanism to amplify displacement which is similar to a slider-crank mechanism. Scire and Teague ${ }^{2}$ developed a PZT stage with a travel of $50 \mu \mathrm{m}$ and subnano resolution. Lloyd and Quate $^{3}$ designed a stage operated at temperature of $4.2 \mathrm{~K}$ with 10 $\mu \mathrm{m}$ travel range. Kusakari and Yoshikawa ${ }^{4}$ designed a three degree-of-freedom mechanism which has an amplifying gain of 3. Physic Instrumente ${ }^{5}$ provides products of micropositioning stages to meet different applications.

This article presents a new optimized design configuration of a micropositioner mechanism with a large travel range. The linear translation mechanism combines a PZT actuator with linear motion device to achieve a travel range over $100 \mu \mathrm{m}$. The PZT actuator, having a size of $5 \mathrm{~mm} \times 5 \mathrm{~mm} \times 20 \mathrm{~mm}$, is capable of traveling $11.6 \mu \mathrm{m}$ and generating a force of $567 \mathrm{~N}$ at $100 \mathrm{~V}$. The linear motion device incorporates two linkages to achieve the requirement. One is a multiple Scott-Russell linkage to magnify the travel of PZT to the desired range. The other is a parallel guiding linkage forming by leaf springs to minimize lateral and an-

${ }^{a)}$ Electronic mail: shchang@ccms.ntu.edu.tw gular deviations. Both linkages utilize flexure hinges to avoid backlash, stick-slip friction, and the need of lubricants.

The design of this mechanism is accompanied by mathematical models based on finite element simulation using a commercial software ANSYS. In addition, this article applies quality engineering techniques to optimize the geometry and the configuration of the linear motion device, and to achieve the goal of maximum mechanical displacement gain and minimum lateral displacement. The stage of the optimized design is made with flexure hinges and linkages which are wire electro-discharge machined (EDM) from a blank of 6061-T6 aluminum. In experiments, the actual travel displacement is measured by a laser interferometer and dynamic characteristics are measured by a spectrum analyzer.

Thus, this article contributes a new design configuration which is useful in stimulating ideas. The application of quality engineering is also useful to new engineers.

\section{DESIGN AND ANALYSIS}

\section{A. Basic mechanism}

A linkage used in the micropositioning stage is the Scott-Russell mechanism. ${ }^{6}$ This mechanism is shown in Fig. 1 where $O$ is the fixed pin joint, $M$ is a pin connected link $\overline{O M}$ and $\overline{A P}$, and point $A$ moves along the $x$ axis. Assume $\overline{O M}=\overline{A M}=\overline{P M}=1, \angle O A M=\theta$, then the coordinates of point $P$ are

$$
\begin{aligned}
& x_{P}=0, \\
& y_{P}=\sqrt{41^{2}-x_{a}^{2}},
\end{aligned}
$$

where $x_{p}$ and $y_{p}$ are the $x$ and $y$ coordinates of point $P$, respectively, and $x_{a}$ is the $x$ coordinate of point $A$.

Thus, under condition $\overline{O M}=\overline{A M}=\overline{P M}$, the ScottRussell mechanism gives point $P$ an exact straight-line motion along the $y$ axis when point $A$ moves along the $x$ axis. Besides, the relations between the $x$ coordinate of point $A$ and $y$ coordinate of point $P$ is 


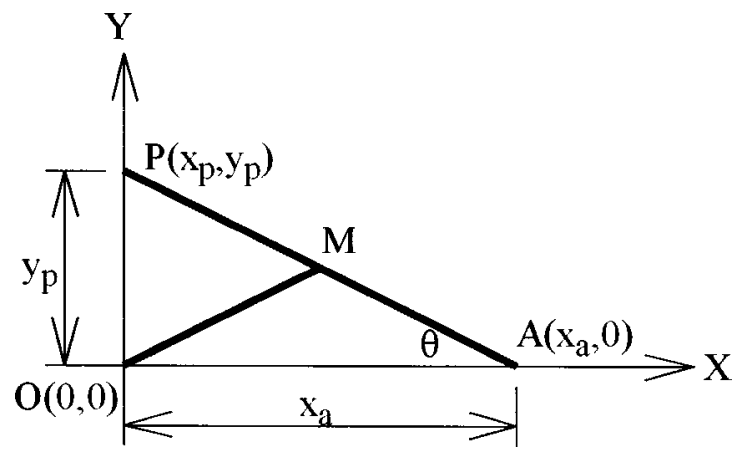

FIG. 1. A Scott-Russell mechanism. Point $O$ is fixed and point $M$ is hinged. The $x$-axis motions of point $A$ are converted into $y$-axis motions of point $P$.

$$
x_{a}^{2}+y_{P}^{2}=\left(x_{a}+\Delta x_{a}\right)^{2}+\left(y_{P}+\Delta y_{P}\right)^{2},
$$

where $\Delta x_{a}$ is the change of $x$ coordinate of point $A$ and $\Delta y_{p}$ is the change of $y$ coordinate of point $P$. Solving this equation to determine $\Delta y_{p}$ from $x_{a}, y_{p}$, and displacement $\Delta x_{a}$, we find that

$$
\Delta y_{P}=-y_{P}+\sqrt{y_{P}^{2}-2 x_{a} \Delta x_{a}-\Delta x_{a}^{2}} .
$$

Thus the relation between displacement $\Delta x_{a}$ and $\Delta y_{p}$ is nonlinear, but when $\Delta x_{a} \rightarrow 0$, this relation can be written as

$$
\frac{\Delta y_{P}}{\Delta x_{a}} \cong \frac{d y_{P}}{d x_{a}}=\frac{-x_{a}}{\sqrt{41^{2}-x_{a}^{2}}}=\frac{-x_{a}}{y_{P}}=-\tan \theta .
$$

Thus, when point $A$ moves with a small displacement $\Delta x_{a}$ along the $x$ axis, point $P$ will move along the $y$ axis and the displacement of point $P$ equals $\tan \theta$ times $\Delta x_{a}$. From this displacement relation, the Scott-Russell mechanism, can be used as a straight-line mechanism with fixed amplifying factor in the field of precision micropositioning.

It is difficult to obtain a large amplifying factor with a single stage of Scott-Russell linkage. Thus, the multiple Scott-Russell mechanism is designed in this article to achieve large travel range.

\section{B. Micropositioning stage}

The schematic representation of the whole micropositioning stage is shown in Fig. 2. The arrows in the figure indicate the motion of components in the stage. The goal of the micropositioning stage is to provide a precision linear positioning mechanism with large travel range. The stage consists of an amplifying device and a parallel leaf spring guiding device. The amplifying device used to amplify the length changes of the piezoelectric actuator, component 1 , is composed of a multiple Scott-Russell mechanism which is indicated by components 2 and 3 in the figure. The parallel leaf spring guiding device consisting of leaf springs is to guide the motion of the stage platform and to avoid the lateral displacements and angular deviations. The flexure hinges fabricated with a single metal blank can avoid the backlash, stick-slip friction, and the need for lubricants. Components 1-5 are connected in sequence to transfer the motion from the actuator to the platform.

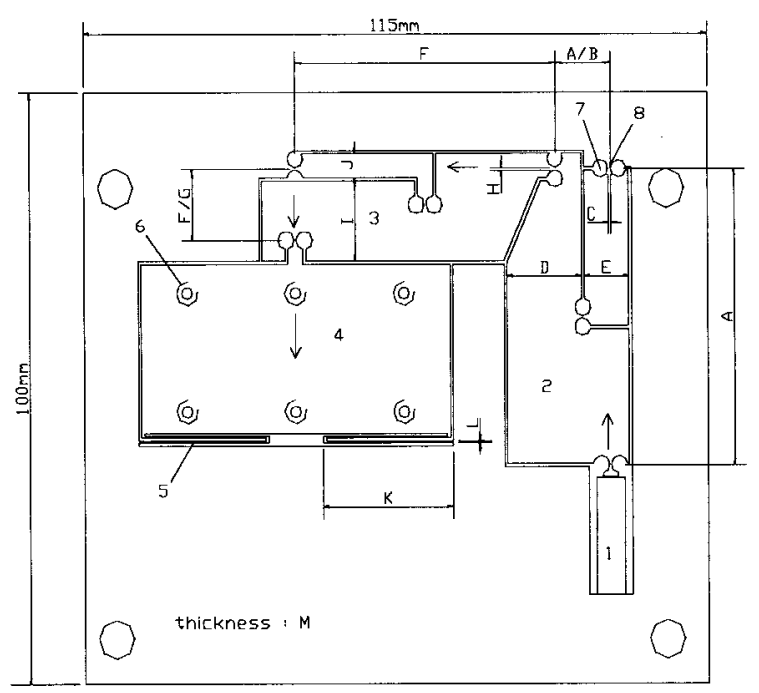

FIG. 2. Schematic representation of the micropositioning stage. (1) PZT, (2) the first linkage, (3) the second linkage, (4) platform, (5), leaf springs, (6) tapped hole, (7) cut-out slot to form flexure hinges, (8) flexure hinges. The key dimensions are represented by the control factors $A$ to $M$. The arrows in the figure indicate the motion of components in the stage.

The motion of the components in the micropositioning stage is described as follows. The length of the piezoelectric actuator, component 1 , changes due to the driving voltage between the electrodes of the piezoelectric actuator. This length change pushes or pulls the input terminal of the first stage of Scott-Russell linkage, component 2, and causes displacements of the output terminal of the linkage. Similarly, the second stage of Scott-Russell linkage, component 3, is driven by the output displacements of component 2. Finally, the platform, component 4 , moves due to the output motion of the second linkage. The leaf springs, component 5, joined to the platform can offer reaction force to prevent the platform from rotating and moving laterally. The two stages of Scott-Russell linkage are designed to amplify their input displacements so that the derived travel range of the platform is significantly larger than the range of length changes of the actuator.

\section{FEM analysis}

In this article, the finite element method (FEM) was used to analyze the performance of the micropositioning stage, such as the displacement, natural frequency, and vibration mode. In this case, the thickness of the stage is relatively large if compared with other critical dimensions of the micropositioning stage, such as thickness of the flexure hinges. Thus the stage is so thick that the motion of the stage will be in a plane perpendicular to the thickness of the stage and the out-of-plane behavior is negligible. The two dimensional simulation is adequate to analyze a plane problem. The micropositioning stage was modeled into approximately more than thousands of finite elements which include the two dimensional solid element named PLANE82 in ANSYS. This element is defined by eight nodes having two degrees of freedom at each node which are translations in the $x$ and $y$ 
directions. It provides more accurate results for irregular shapes. The geometry of the model is based on a design similar to Fig. 2. The element sizes are different for each part of the stage and are about a quarter of the minimum dimension of the local region. The holes at the corners of the stage are fixed to the ground to simulate the effects of the fasteners. One end of the piezoelectric actuator was given a displacement as the excitation source to the micropositioning stage. Theoretically, the maximum stress of the stage is expected to happen at flexural hinges. However, to ensure the safety of the stage, the maximum stress of the whole stage was displayed and checked not to exceed the allowable stress of the 6061-T6 aluminum.

The results of finite element analysis, such as the displacements and the lateral motion of the center of the platform, were observed and recorded. The data were further used by the optimization technique to find an optimized configuration of the micropositioning stage.

\section{OPTIMUM DESIGN}

The overall design problem is to optimize the geometry and configuration of the flexure hinges and the Scott-Russell linkages to achieve the goal of maximum mechanical displacement gain and minimum lateral displacement while taking into account the demands imposed by stretching and bending of flexure hinges and linkages. The Taguchi method for quality engineering ${ }^{7}$ provides the capability of solving this design problem. It has been successfully applied in the optimization of output power of a $\mathrm{CO}_{2} \operatorname{laser}^{8}$ and in fuzzy control. ${ }^{9}$

The Taguchi method evolves from statistically designed experiments developed by R. A. Fisher in the 1920s. It is a method of optimization for performance, quality, and cost (parameter design and quality loss function). It also analyzes a product or process and identifies the solutions which are minimally sensitive to causes of variations. The Taguchi method uses orthogonal arrays to study behaviors of many variables from the minimum amount of test data and experiments.

The Taguchi method was extensively used during the parameter design stage, in which the optimal level of each design factor or parameter will be determined. Two types of factors which affect performance of the micropositioning stage were identified: control factors and noise factors. Control factors can easily be controlled such as, in this case, the geometry and configuration of the flexure hinges, the ScottRussell linkages, and the leaf springs. Noise factors are those that are difficult or expensive to control, e.g., external vibration and atmosphere temperature.

Control factors can be categorized into two main groups: variability-control factors and target-control factors. Variability-control factors or "control" factors are those that affect the variability in the output or response. Target-control factors or "signal" factors are those that affect only the main level of a response. Frequently, however, a control factor may affect both the main level and the variability of the response.

Noise factors can be classified into three groups: external noise, internal noise, and product-to-product noise. External noises are caused by the variations in measurement and environmental conditions. Internal noises are variations caused by the aging process or deterioration of the internal elements. Product-to-product noises are variations caused by manufacturing imperfections. Statistically, noise factors cause the output response to deviate from the target values in the nearrandom fashion.

The strategy of the Taguchi method is to recognize the existence of control factors and noise factors, and to treat them separately. To handle them, the Taguchi method utilizes orthogonal arrays. The major steps of implementing the Taguchi's parameter design are: (1) identify the factors of interest; (2) identify the levels of each factor; (3) select the appropriate orthogonal array; (4) assign the factors to columns; (5) conduct the experiment and/or analysis; (6) analyze the data and determine the optimum level of each factor; and (7) conduct the confirmation experiment and/or analysis. The confirmation experiment is important as it verifies the conclusions. If the results are not confirmed or unsatisfactory, additional experiments or analysis may have to be conducted.

Hence, the essence of the Taguchi method is to find the optimal values of the control factors (e.g., design variables of the stage) by using orthogonal arrays and analysis of variance (ANOVA). In this case, it was the FEM results instead of the experimental results that were to be used to analyze the performance of stage at a different setting of design factors.

A total of 13 control factors respectively denoted by $A, B, \ldots, M$ were used in this case. Among them, five control factors were selected for each Scott-Russell linkage, and two for the parallel leaf springs. For the Scott-Russell linkages, the total length, the amplifying factor, and the width of flexure hinges and two links in the Scott-Russell linkage were chosen as the control factors. The width of flexure hinges for each Scott-Russell linkage is denoted by control factors $C$ and $H$, respectively. Thus the width of flexure hinges in one Scott-Russell linkage is the same but may be different between distinct Scott-Russell linkages. The width of the flexure hinge between the second Scott-Russell linkage and the output stage is the same as those of the second Scott-Russell linkage. Control factors for parallel leaf springs are the length and width of springs. The last control factor is the thickness of the micropositioning stage. Three levels were selected for each control factor. The 13 control factors are shown in Fig. 2 and also listed in Table I with the selected levels. The amplifying factor, one of the chosen control factors, is defined as the ratio of the output displacement to the input displacement of each stage of ScottRussell linkage. From Eq. (5), the amplifying factor $f$ is

$$
f=\frac{x_{a}}{y_{P}},
$$

where the physical meaning of $x_{a}$ and $y_{p}$ are shown in Fig. 1. The ranges of levels for each control factor were chosen to meet the physical and fabrication constraints. For example, the minimum width of the flexure hinges and leaf springs is constrained by the resolution of precision wire cutting used 
TABLE I. Control factors and levels. The optimum setting is shown in bold style. All factors are in units of mm, except that $B$ and $G$ are dimensionless.

\begin{tabular}{llcccc}
\hline \hline & & \multicolumn{3}{c}{ Level } \\
\cline { 3 - 6 } & & & 1 & 2 & 3 \\
\hline 1st & total length & $A$ & $\mathbf{5 0}$ & 55 & 60 \\
linkage & amplifying factor & $B$ & 4.0 & 4.5 & $\mathbf{5 . 0}$ \\
& width of flexure hinge & $C$ & 0.3 & $\mathbf{0 . 4}$ & 0.5 \\
& width of link 1 & $D$ & 11.5 & 12.5 & $\mathbf{1 3 . 5}$ \\
& width of link 2 & $E$ & $\mathbf{8 . 5}$ & 9.5 & 10.5 \\
2nd & total length & $F$ & 40 & 44 & $\mathbf{4 8}$ \\
linkage & amplifying factor & $G$ & $\mathbf{4 . 0}$ & 5.0 & 6.0 \\
& width of flexure hinge & $H$ & $\mathbf{0 . 3}$ & 0.4 & 0.5 \\
& width of link 1 & $I$ & 11.5 & 12.5 & $\mathbf{1 3 . 5}$ \\
& width of link 2 & $J$ & $\mathbf{4 . 0}$ & 5.0 & 6.0 \\
\multirow{2}{*}{ Spring } & length & $K$ & 20 & 22 & $\mathbf{2 4}$ \\
& width & $L$ & $\mathbf{0 . 3}$ & 0.4 & 0.5 \\
\multirow{2}{*}{ Thickness of whole stage } & $M$ & $\mathbf{1 0 . 0}$ & 12.5 & 15.0 \\
\hline \hline
\end{tabular}

to fabricate the stage. In addition, during the fabrication, the existing side force between the wire and the metal blank and residual stress may result in serious distortion of the fabricated stage. To avoid the distortion, some dimensions of the stage must be constrained. Although the bending of the links in the multiple Scott-Russell linkage was simulated in the FEM model, the width of the links was in practice chosen to be larger than the thickness of the flexure hinges. This constraint causes the bending of flexure hinges to be much larger than that in the links, and hence the general relationship of Scott-Russell linkage can be maintained.

The 13 factors were assigned to a standard L27 orthogonal array which is the most possible economic array. The L27 orthogonal array contains 27 different level settings of control factors. FEM analyses were carried out to solve the displacement for each setting in the L27 orthogonal array.

ANOVA was carried out on the FEM results to determine whether the control factors had any statistically significant effects on the displacement sensitivity. The average values for levels of control factors are computed by

$$
A_{i j}=\frac{1}{n}\left(\eta_{i j 1}+\eta_{i j 2}+\cdots+\eta_{i j n}\right),
$$

where $A_{i j}$ represents average value for the $i$ th level of the $j$ th control factor, $\eta_{i j k}$ is one of FEM results whose row contains the $i$ th level at the $j$ th column in orthogonal array, and $n$ is the total number of rows containing the $i$ th level at the $j$ th column.

A computer program was written using Microsoft EXCEL to implement the procedure of ANOVA. Figure 3 shows the results of ANOVA for each control factor. In the figure, symbols a and $\mathrm{b}$, respectively, denote the computed displacements and lateral displacements of the output member of the stage. Each subplot contains three data points which correspond to the average values of levels of control factors. After completion of all trials and the data were analyzed, the recommended optimum levels of the control factors can be found and denoted by bold-style fonts in Table I. The final optimized design of the stage is hereby finally determined.
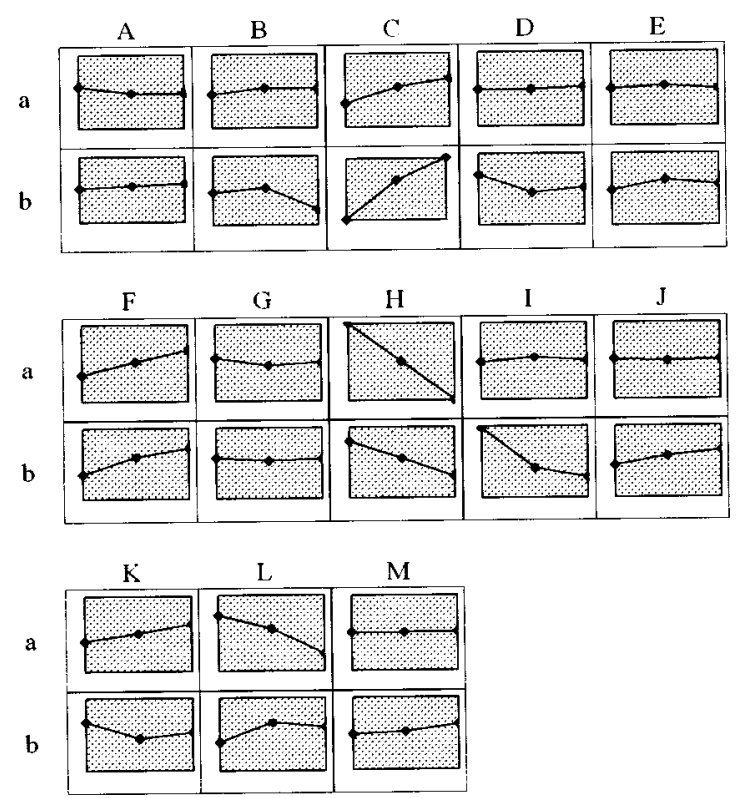

FIG. 3. Signal-to-noise (S-N) ratios for each control factor. Symbols a and $\mathrm{b}$, respectively, denote the computed displacements and lateral displacements of the output member of the stage. The ordinate and abscissa of each subplot are displacements and levels respectively.

\section{STAGE FABRICATION}

Fabricating the stage involves two steps: machining the flexure hinges and linkages from a metal blank, and installing the piezoelectric actuator into the flexure-pivot linkage. To make the stage compact and to utilize the advantages of monolithic construction, the flexure hinges and levers were formed in one piece of metal. This configuration eliminates the bulk and complexity of fasteners, and essentially no tolerance exists.

The flexure-pivot linkage was machined from a 6061-T6 aluminum metal blank with dimension of $115 \times 100$ $\times 10 \mathrm{~mm}$. Machining the flexure-pivot linkage consists of precision wire electro-discharge machining (EDM) the metal blank to form the desired shape, the flexure hinges and slots in the blank to form the linkages, and a cutout to accept the piezoelectric actuator. Two relatively critical operations were involved. Both the moment required to bend the flexure hinges and the force to stretch were sensitive to the thickness of the flexure hinge. Therefore a thickness tolerance of $\pm 20 \mu \mathrm{m}$ was chosen and it required the tolerance of a hole location within $\pm 10 \mu \mathrm{m}$. The second operation was to form the cutout to accept the piezoelectric actuator. The two surfaces of the cutout for accepting the actuator should be ensured to be as flat and parallel as possible. Nonflatness and nonparallelism were both critical to the undesired angular motion of the stage.

A multilayer piezoelectric ceramics with dimension 5 $\times 5 \times 20 \mathrm{~mm}$ was installed in the flexure-pivot linkage by fitting the actuator into the cutout of the metal blank and then bonding in place. A photograph of the micropositioning stage is shown in Fig. 4.

\section{EXPERIMENT AND MEASUREMENT RESULTS}

The experimental setup was shown in Fig. 5. The characteristics of the output member of the stage, such as the 


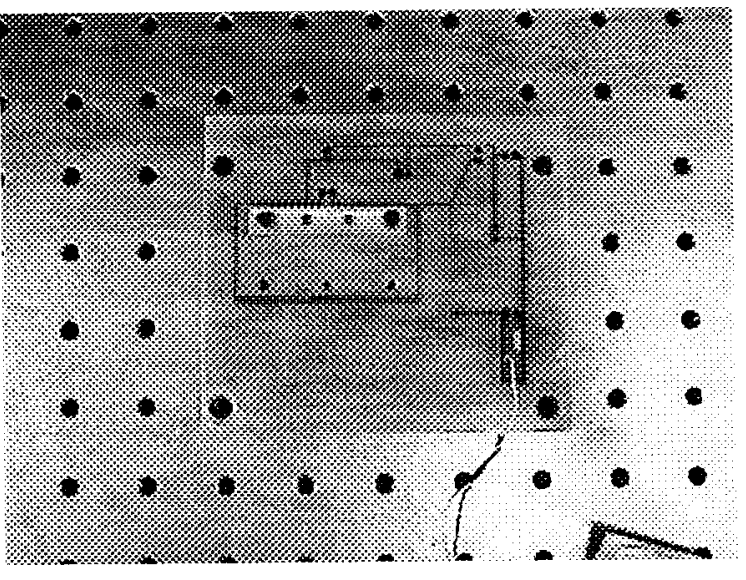

FIG. 4. Photograph of the micropositioning stage.

displacement and the angular deviation, were measured by a Polytec laser interferometer which has a displacement resolution of $8 \mathrm{~nm}$ and a bandwidth of $50 \mathrm{kHz}$. A fine-grained scatter-reflected paper was pasted on the output member in the appropriate plane to enhance the optical reflection of the incident laser beam for the measurement of the output member's motion. The function generator and a personal computer with a programmable digital-to-analog card were used to generate the desired wave form signal for testing the stage. Generally, the function generator provided periodical sinusoidal or step wave forms while the computer generated signals with special forms, such as stairway-type signals and impulse. The signals generated by the function generator or personal computer were amplified by a power amplifier to drive the piezoelectric actuator installed in the stage. The oscilloscope and dynamic signal analyzer were used to display, store, and analyze the experimental results in both time and frequency domains. The tested stage was placed on an air-bearing supported optical table and isolated from external vibrations.

\section{A. Open-loop measurement}

From the experiments, the observed maximum output displacements were approximately $112 \mu \mathrm{m}$ for an applied voltage of $100 \mathrm{~V}$. Thus, the output displacement sensitivity was $1.12 \mu \mathrm{m} / \mathrm{V}$. Maintaining a constant focusing condition in the optical microscopes is an essential requirement. The

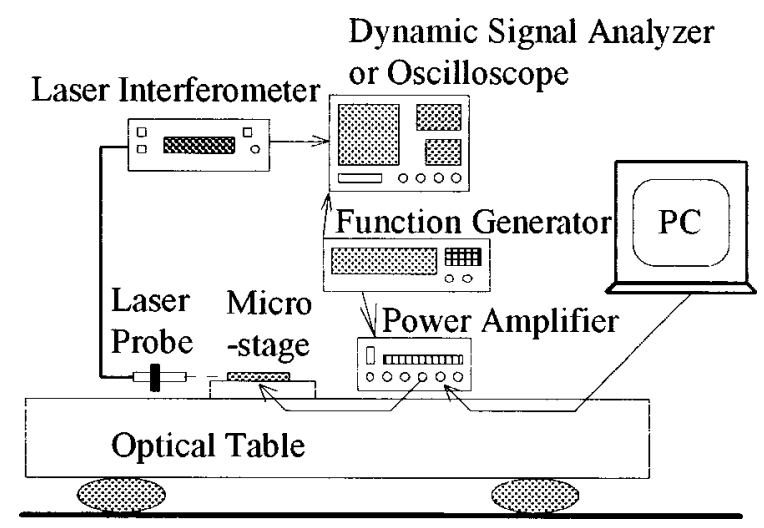

FIG. 5. Experimental setup.

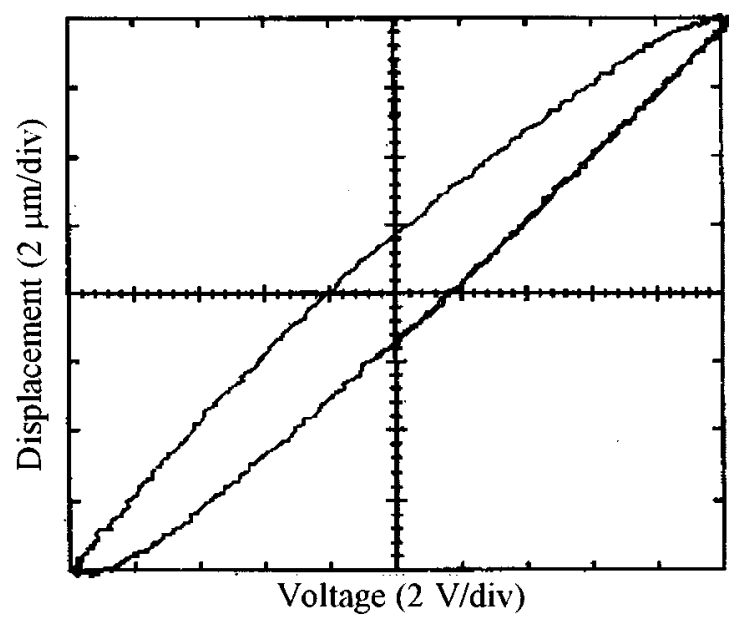

FIG. 6. The measured hysteresis curve without controller. The $x$ axis is the driving voltage of the piezoelectric actuator. The $y$ axis is the displacement of the output member of the stage.

angular deviation characteristics of the stage's output member were therefore measured. Two points on the output member were chosen to measure their displacements by two laser beams of the same phase at the same time. The phase difference between laser beams caused by motion of the output member thus was analyzed by the Polytec interferometer and transformed to the corresponding angular displacement between two measurement points. From the experiments, the angular motion of the stage was found to be less than 0.278 $\mu \mathrm{rad}$ at output displacements of $1 \mu \mathrm{m}$ at low excitation frequency. It is equivalent to a maximum angular deviation of $31.136 \mu \mathrm{rad}$ at maximum output displacements of $112 \mu \mathrm{m}$.

Smooth linear output displacements as a function of the applied voltage were expected. Figure 6 shows measured displacements as a function of the voltage applied to the piezoelectric element. In the figure, the signal of channel 1 was the sinusoidal driving voltage wave of the piezoelectric actuator produced by the function generator. The signal of channel 2 was the displacement of the output member of the stage measured by the laser interferometer at a sensitivity of $20 \mu \mathrm{m} / \mathrm{V}$. The results demonstrate that the hysteresis in the stage's output gives an offset of approximately $22.5 \%$ of the maximum displacement.

\section{B. Open-loop compensation}

To reduce the hysteresis of the piezoelectric-actuated stage, several closed loop control methods, such as strain gage feedback and self-sensing techniques, were used in many articles. In this article, a simple open loop technique similar to Ref. 10 was used. This open loop controller is able to compensate the phase lag inherent in the stage. It used a phase-lead compensation analog circuit that requires no feedback from the output member of the stage. Thus the whole controlled system can be simple. The phase-lead compensation circuit was placed after the power amplifier, and the signal of function generator was the command signal without feedback control. The transfer function of the circuit can be expressed as a function of an independent frequency parameter $s$ : 


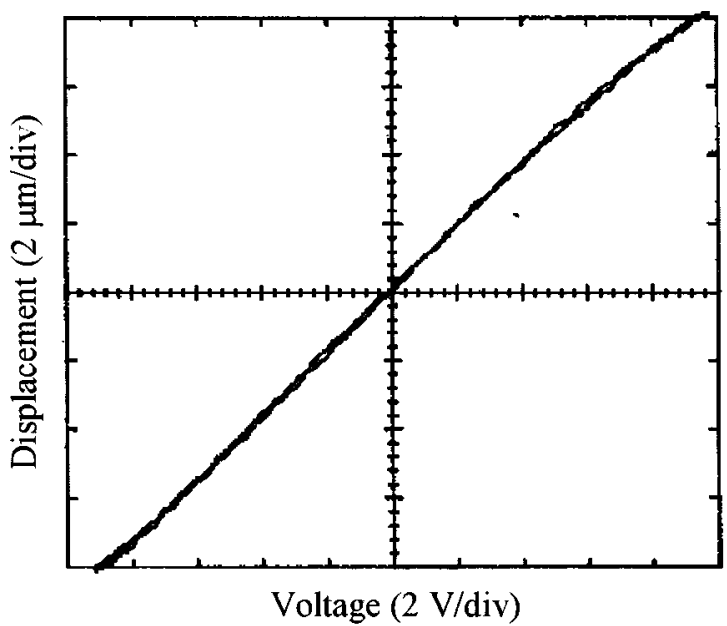

FIG. 7. The measured hysteresis curve with controller. The $x$ axis is the driving voltage of the piezoelectric actuator. The $y$ axis is the displacement of the output member of the stage.

$$
T(s)=\frac{A s+1}{B s+1} .
$$

The parameters $A$ and $B$ were tuned and chosen so as to cancel out the frequency-dependent phase lag of the stage over a low excitation frequency range. The hysteresis of the stage after the open-loop compensation, shown in Fig. 7, was reduced significantly if compared with Fig. 6. In addition, from Fig. 7, an output displacement sensitivity of $0.86 \mu \mathrm{m} / \mathrm{V}$ was observed for the micropositioning stage after open-loop compensation.

For a precision positioning device, a wide dynamic operating range is needed. The dynamic signal analyzer was used to proceed the frequency response function measurement for the output displacement of the stage. The command signal was the sweep sinusoidal excitation produced by the dynamic signal analyzer itself. The output signal of the interferometer was used to represent the displacement of the stage at the chosen sensitivity of $80 \mu \mathrm{m} / \mathrm{V}$. Figure 8 is the frequency response function of the stage without any addi-

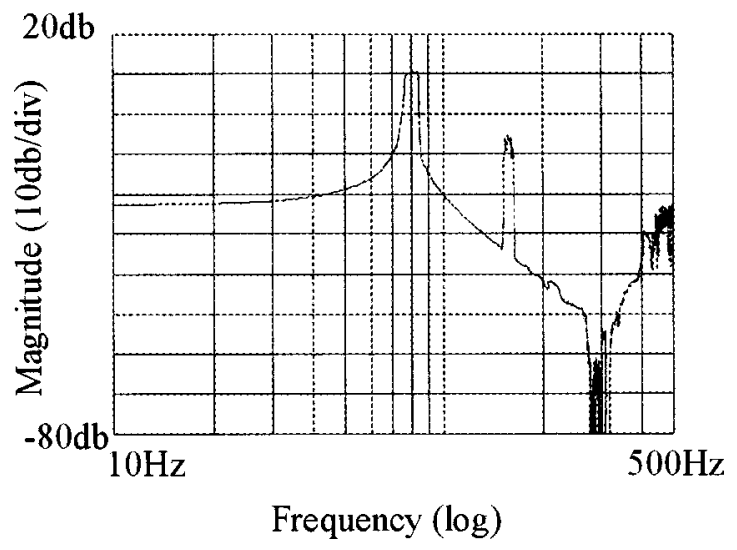

FIG. 8. The measured frequency response function of the micropositioning stage. The ordinate is the vibration amplitude of the output member of the stage. The abscissa is the excitation frequency of the driving voltage applied to the piezoelectric element.

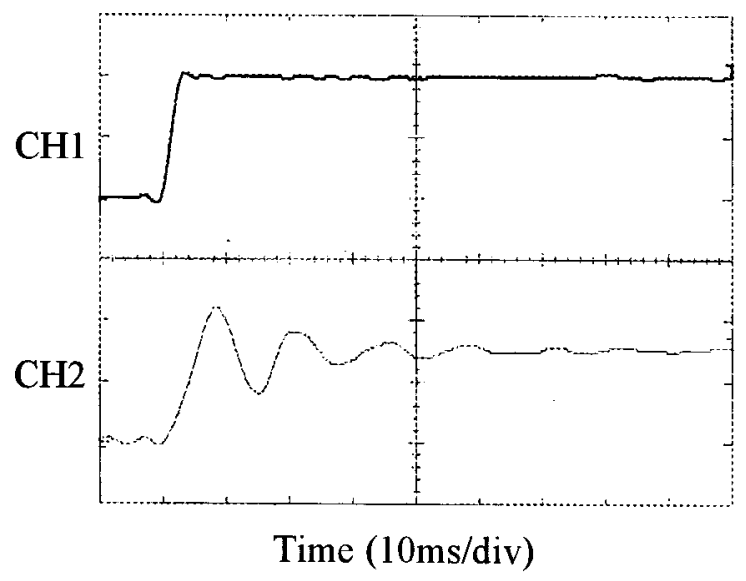

FIG. 9. The step response of the micropositioning stage. $\mathrm{CH} 1$ is the driving voltage of the piezoelectric element $(5 \mathrm{~V} / \mathrm{div}) . \mathrm{CH} 2$ is the displacement of the output member of the stage $(8 \mu \mathrm{m} / \mathrm{div})$.

tional controller. From the frequency response function, a first natural frequency of $80 \mathrm{~Hz}$ was observed.

In addition, the dynamic response of the stage should settle quickly, for the stage can be isolated from external vibrations, and reach the steady state in a short time when the command signal changes suddenly. The step response of the output member of the stage is shown in Fig. 9. Channel 1 is the command signal and channel 2 is the response of the output member detected by the laser interferometer. From this figure, the settling time of the stage is approximately 50 ms at the $10 \%$ full travel range.

To find the finest resolution of the stage, a stairway-type command signal is used to drive the piezoelectric actuator of the stage. The applied voltage of each stairway is attenuated in sequence to see if the response of the stage can be distinguished. A typical response of the stage under the excitation of a periodic stairway-type signal (the upper curve) is shown (the lower curve) in Fig. 10. From experiments, the smallest resolution of the displacement of the stage is $0.04 \mu \mathrm{m}$.

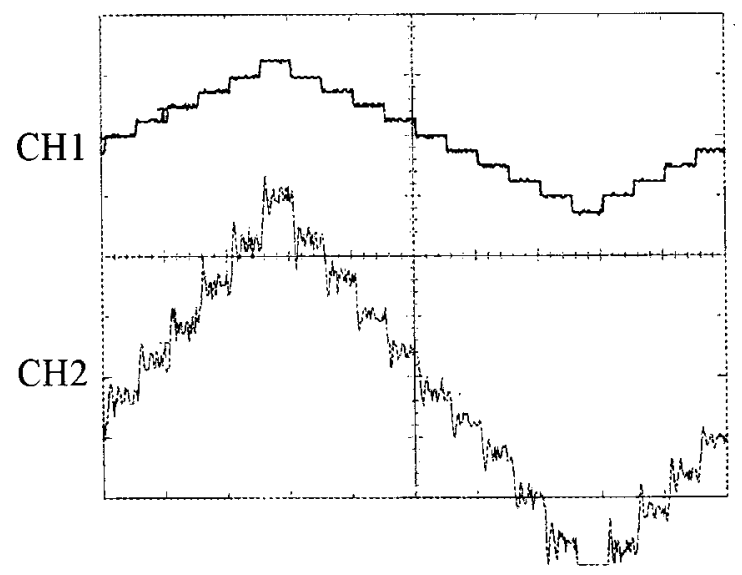

Time $(100 \mathrm{~ms} / \mathrm{div})$

FIG. 10. The stage's displacement response to the stairway-type input signal. $\mathrm{CH} 1$ is the driving voltage of the piezoelectric element $(2 \mathrm{~V} / \mathrm{div}) . \mathrm{CH} 2$ is the displacement of the output member of the stage $(0.04 \mu \mathrm{m} / \mathrm{div})$. 


\section{DISCUSSION}

Careful examination of the design will see two stages of Scott-Russell mechanism used to amplify the length changes of the piezoelectric actuator. According to the results of the optimum design, devices which have been built had design gains of 5.0 for the first mechanism and 4.0 for the second mechanism, which should have produced a total gain of 20.0 theoretically. The flexure hinge, with thicknesses of $0.4 \mathrm{~mm}$ for linkage 2 , and $0.3 \mathrm{~mm}$ for linkage 3 , is formed by two cut-out holes of radius $1.5 \mathrm{~mm}$. The anticipated input to the system from the piezoelectric actuator for an applied voltage of $100 \mathrm{~V}$ was $11.6 \mu \mathrm{m}$ to produce output displacements of about $232 \mu \mathrm{m}$. However, the observed outputs for this voltage have been only approximately $112 \mu \mathrm{m}$. Thus, the observed gain is approximately 9.66 . That is due to the angular deformation existing in the flexure hinges and the reaction force produced by the leaf springs which reduces the length changes of the piezoelectric actuator. In addition, the bending of the linkages also decreases the output displacement of the stage.

\section{ACKNOWLEDGMENT}

This work was supported by the National Science Council, Taiwan, Republic of China, under Grant No. NSC862221-E-002-096.

${ }^{1}$ E. Furukawa, M. Mizuno, K. Terada, Int. J. Jpn. Soc. Precis. Eng. 25, 315 (1991).

${ }^{2}$ F. E. Scire and E. C. Teague, Rev. Sci. Instrum. 49, 1735 (1978).

${ }^{3}$ L. J. L. Comb, Jr. and C. F. Quate, Rev. Sci. Instrum. 59, 1906 (1988).

${ }^{4}$ T. Kusakari and M. Yoshikawa, Jpn. Soc. Precis. Eng. 62, 130 (1996).

${ }^{5}$ Physik Instrumente, "Products for micropositioning," Catalogue Edition E.

${ }^{6}$ G. H. Martin, Kinematics and Dynamics of Machines (McGraw-Hill, New York, 1982), p. 52.

${ }^{7}$ P. J. Ross, Taguchi Techniques for Quality Engineering (McGraw-Hill, New York, 1989).

${ }^{8}$ S. C. Tam, Y. M. Noor, and L. J. Yang, IEEE J. Quantum Electron. 29, 192 (1993).

${ }^{9}$ P. J. King and E. H. Mamdani, Automatica 13, 235 (1977).

${ }^{10}$ K. Duong and E. Garcia, J. Intell. Mater. Syst. Struct. 6, 292 (1995). 